\title{
Correction to "Amlexanox Inhibits Cerebral Ischemia-Induced Delayed Astrocytic High-Mobility Group Box 1 Release and Subsequent Brain Damage"
}

In the above article [Halder SK and Ueda H (2018) J Pharmacol Exp Ther, 365: 27-36; DOI: https://doi.org/10.1124/jpet.117.245340, the reference by Hjelm et al. was incomplete. The complete reference is:

Hjelm F, Tran B, and Fredriksson S (2011) Sensitive detection of cytokines in 1- $\mu$ l serum samples using Proseek®. Nat Methods 8:iii; DOI: 10.1038/nmeth.f.348

The HTML and PDF versions of the article have been corrected. The compositor apologizes for any inconvenience this may have caused. 